\title{
Economic and health care resource utilization burden of central nervous system metastases in patients with metastatic melanoma
}

\author{
Hussein Tawbi, MD, PhD; Karen Bartley, PhD; Arpamas Seetasith, PhD; Matthew Kent, MS; Janet Lee, PhD, PharmD; \\ Elizabeth Burton, MBA; Lauren Haydu, PhD, MPH; and Edward McKenna, PharmD
}

\section{What is already known about this subject}

- Patients with advanced melanoma have a high risk of developing central nervous system (CNS) metastases, and diagnostic brain imaging is recommended as part of staging for patients with metastatic disease.

- Previous cost-estimate studies in patients with melanoma and CNS metastases were conducted before widespread use of targeted therapies and immunotherapies.

\section{ABSTRACT}

BACKGROUND: In patients with metastatic melanoma, central nervous system (CNS) involvement is associated with poor prognosis, increased costs, and higher health care resource utilization (HCRU); however, previous cost-estimate studies were conducted before widespread use of targeted therapies and immunotherapies.

\section{What this study adds}

- Patients with melanoma and CNS metastases incur significantly higher health care resource utilization and costs than those without CNS metastases across inpatient, outpatient, treatment, and pharmacy services; the largest contributors to total costs in the current treatment era are radiology, targeted therapies, and immunotherapies.

- Brain imaging at the time of diagnosis of metastatic melanoma remains underused, and there appears to be an opportunity to improve outcomes through early detection of asymptomatic CNS metastases when they might be more responsive to currently available therapeutic options.

- A substantial proportion of patients require corticosteroids (dexamethasone) for control of symptomatic CNS metastases, have fewer therapeutic options, and represent a population of unmet medical need.

OBJECTIVE: To estimate costs and HCRU in patients with metastatic melanoma with and without CNS metastases in the current treatment era following introduction of targeted therapies and immunotherapies.

METHODS: This real-world retrospective cohort study used data from the IQVIA PharMetrics Plus claims database to estimate and compare costs and HCRU in patients with metastatic melanoma by presence or absence of CNS metastases between

\section{Author affiliations}

Hussein Tawbi, MD, PhD, Melanoma Medical Oncology, The University of Texas MD Anderson Cancer Center, Houston, TX. Elizabeth Burton, MBA, and Lauren Haydu, $\mathrm{PhD}, \mathrm{MPH}$, Department of Surgical Oncology, The University of Texas MD Anderson Cancer Center, Houston, TX. Karen Bartley, PhD; Arpamas Seetasith, PhD; Janet Lee, PhD, PharmD; and Edward McKenna, PharmD, Genentech, Inc., South San Francisco, CA. Matthew Kent, MS, Genesis Research LLC, Hoboken, NJ.

\section{AUTHOR CORRESPONDENCE:}

Hussein Tawbi, 713.792.2121;

htawbi@mdanderson.org

J Manag Care Spec Pharm 2022;28(3):342-53

Copyright $\odot 2022$, Academy of Managed Care Pharmacy. All rights reserved.

January 2011 and June 2019. Patients with at least 2 melanoma claims, at least 2 metastatic claims, and continuous enrollment at least 6 months before and at least 1 month after first metastatic diagnosis were included. Mean per-patient-per-month (PPPM) costs are reported in 2019 US dollars. Analyses were also conducted by time period of first metastatic diagnosis: 2011-2014 (reflecting BRAF inhibitor monotherapy and antiCTLA-4 therapy) and 2015-2019 (reflecting 
availability of BRAF and MEK inhibitor combinations and anti-PD-1/PD-L1 therapies).

RESULTS: Of 4,078 patients, 1,253 (30.7\%) had CNS metastases. Patients with CNS metastases were more likely to receive any treatment $(89.1 \%$ vs $58.9 \% ; P<0.001)$, including systemic treatment $(73.3 \%$ vs $55.4 \% ; P<0.001)$ and radiation $(65.8 \%$ vs $11.8 \% ; P<0.001)$, and to have brain imaging any time after metastatic diagnosis ( $98.3 \%$ vs $67.2 \% ; P<0.001$ ). In patients with CNS metastases, $40.0 \%$ had dexamethasone $4 \mathrm{mg}$ within 30 days of CNS metastatic diagnosis. Patients with CNS metastases incurred higher total mean PPPM costs $(\$ 29,953$ vs $\$ 14,996 ; P<0.001)$. The largest contributors were total radiology $(\$ 2,351$ vs $\$ 1,110)$, targeted therapies ( $\$ 2,499$ vs $\$ 638)$, and immunotherapies $(\$ 7,398$ vs $\$ 5,036)$. HCRU and costs were higher in patients with vs without CNS metastases regardless of time period of first metastatic diagnosis. In patients with CNS metastases, use of any systemic treatment was increased in $2015-2019$ vs $2011-2014$ ( $81.2 \%$ vs $64.5 \% ; P<0.001)$, including chemotherapy $(68.1 \%$ vs $50.0 \% ; P<0.001)$, immunotherapy $(60.9 \%$ vs $30.1 \% ; P<0.001)$, and/or targeted therapies (32.7\% vs $27.4 \% ; P=0.05)$. Mean total PPPM costs for patients with CNS metastases increased from $\$ 28,183$ in 2011-2014 to $\$ 31,569$ in 2015-2019 $(P<0.001)$; main drivers were immunotherapies and targeted therapies.

CONCLUSIONS: CNS metastases occur frequently in patients with metastatic melanoma and are associated with significantly increased economic burden compared with patients without CNS metastases; the largest contributors to total costs in the current treatment era are radiology, targeted therapies, and immunotherapies. Brain imaging remains underused, and there is an opportunity to improve outcomes through early detection of CNS metastases, potentially reducing the high HCRU and costs associated with CNS metastases.

Patients with advanced melanoma have a high risk of developing central nervous system (CNS) metastases. ${ }^{1-3}$ CNS metastases are present at diagnosis in approximately $20 \%-$ $30 \%$ of patients with metastatic melanoma, and up to $50 \%$ of patients develop clinically evident CNS metastases over the course of their disease. ${ }^{3-5}$ Patients with metastatic melanoma and CNS metastases have a poor prognosis, with median survival of 4-6 months. ${ }^{3-5}$ Computed tomography (CT) and magnetic resonance imaging (MRI) are the key imaging modalities used for the diagnosis of CNS metastases. ${ }^{6}$ Because of the high incidence of CNS metastases, diagnostic brain imaging is recommended as part of the staging process for patients with metastatic melanoma but has historically been underused. ${ }^{7-10}$

Treatment for CNS metastases may include high-cost systemic and/or local therapies, including radiation and surgery. ${ }^{11,12}$ Over the last decade, stereotactic radiosurgery has become the primary treatment of choice for patients with CNS metastases ${ }^{13,14}$ but is resource- and time-intensive compared with whole-brain radiation therapy. Over the last decade, the introduction of BRAF and MEK inhibitors (from 2011 for BRAF inhibitor monotherapy and from 2015 for BRAF and MEK inhibitor combination therapy) $)^{15-21}$ and immune checkpoint inhibitors (from 2011 for anti-cytotoxic T-lymphocyte-associated protein-4 [CTLA-4] and from 2015 for anti-programmed death 1 [PD-1] $)^{22-25}$ has expanded treatment options and dramatically improved survival outcomes for patients with metastatic melanoma. These novel agents have also demonstrated intracranial activity ${ }^{26-33}$; however, this activity appears to be limited to patients with asymptomatic or low-burden CNS metastases. ${ }^{26-30}$ Dexamethasone is the corticosteroid of choice for temporary symptomatic relief of CNS metastases ${ }^{34}$; however, its use may preclude other therapeutic options or limit their effectiveness, particularly immunotherapy. ${ }^{35}$

CNS involvement adversely impacts patient quality of life and is associated with increased costs and higher levels of health care resource utilization (HCRU). ${ }^{1,4,36}$ Real-world studies estimating the cost of care can help inform treatment decisions for payers and clinicians. However, previous cost-estimate studies in patients with melanoma and CNS metastases were conducted before widespread use of targeted therapies and immunotherapies. ${ }^{36}$ Therefore, we conducted a real-world study to estimate costs and HCRU in patients with metastatic melanoma with and without CNS metastases in the current treatment era following introduction of targeted therapies (BRAF and MEK inhibitors) and immunotherapies (immune checkpoint inhibitors).

\section{Methods}

\section{STUDY DESIGN AND PATIENTS}

This retrospective cohort study used data from the IQVIA PharMetrics Plus longitudinal claims database, which contains adjudicated claims data for more than 190 million commercially insured individuals throughout the United States. Patients were included if they had at least 2 claims with a diagnosis code for melanoma at least 30 days apart between January 1, 2011, and June 30, 2019; had at least 2 claims with a diagnosis code for metastatic cancer over the same time period; were aged at least 18 years at first metastatic diagnosis date; and had continuous enrollment for at least 6 months before and at least 1 month after their first metastatic diagnosis date.

Patients were excluded if they had metastatic cancer within 6 months before the first metastatic diagnosis date, evidence of another primary cancer within 6 months before 
the first metastatic diagnosis date (except for localized basal cell and squamous cell carcinomas requiring no surgical intervention during the study period), clinical trial enrollment at any time starting 6 months before the first metastatic diagnosis date, or evidence of regional lymph node metastases only.

Melanoma diagnosis was identified using International Classification of Diseases, Ninth Revision, Clinical Modification (ICD-9-CM) code 172.x or International Classification of Diseases, Tenth Revision, Clinical Modification (ICD-10-CM) codes C43x or D03x. Metastatic cancer was identified using ICD-9-CM codes 196.xx-198.xx or ICD-10-CM codes $\mathrm{C} 77 \mathrm{x}-\mathrm{C} 79 \mathrm{x}$. Patient cohorts were defined by the presence or absence of diagnosis of CNS metastases, identified using the ICD-9-CM code 198.3 or ICD-10-CM code C79.31.

The first cohort included patients with a diagnosis of CNS metastases at any time from the time of metastatic diagnosis to the end of follow-up. The second cohort included patients with no diagnosis of CNS metastases at metastatic diagnosis or at any time during the follow-up period and no claims for brain surgery.

The index date was defined as the date of the first claim with a diagnosis of metastatic cancer, indicating first diagnosis of metastatic melanoma (Supplementary Figure 1, available in online article). Patients were followed from the date of first diagnosis of metastatic melanoma until the end of continuous enrollment in a health plan or the end of the study period, whichever occurred first.

Data were deidentified in compliance with the Health Insurance Portability and Accountability Act (HIPAA) and institutional review board approval was not required.

\section{OUTCOME MEASURES}

Outcomes of interest were the proportion of patients with CNS metastases; clinical characteristics associated with CNS metastases; HCRU (ie, hospitalization, emergency department visits, and melanoma treatments); costs (ie, total cost of care, cost of hospitalizations, cost of outpatient care excluding melanoma treatments, cost of melanoma treatments, and pharmacy costs excluding melanoma treatments); diagnostic brain MRI/CT at the time of first metastatic diagnosis and at the time of first CNS metastasis diagnosis; and dexamethasone prescription at the time of first CNS metastasis diagnosis.

Costs included third-party payer costs and out-ofpocket patient costs. Costs were calculated as per patient per month (PPPM), adjusted to 2019 US dollars using the Consumer Price Index for Medical Care Services. HCRU was evaluated over the follow-up period, with no adjustment for differences in follow-up duration.

\section{ANALYSES}

Outcomes were compared between the cohort of patients with metastatic melanoma and a diagnosis of CNS metastases and the cohort of patients with metastatic melanoma without a diagnosis of CNS metastases identified between 2011 and 2019. In addition, for patients with CNS metastases, costs were also compared between the periods before and after CNS metastatic diagnosis (pre-CNS metastatic period vs post-CNS metastatic period). The pre-CNS metastatic period was defined as the time from the metastatic melanoma diagnosis date until 30 days before the diagnosis of CNS metastases. The post-CNS metastatic period was defined as the time from 30 days before the diagnosis of CNS metastases until the end of follow-up. The post-CNS metastatic period included the 30 days before diagnosis of CNS metastases in order to capture costs of diagnostic tests commonly performed before the final diagnosis of CNS metastases.

Separate analyses were also conducted according to the time period of diagnosis to understand the impact on costs of modern treatments (targeted therapies and immunotherapies) and any other changes over time. The first cohort included patients with a first diagnosis of metastatic melanoma between January 1, 2011, and December 31, 2014 (period 1), during which time systemic therapies predominantly comprised BRAF inhibitor monotherapy and anti-CTLA-4 therapy. The second cohort included patients with a first diagnosis of metastatic melanoma between January 1, 2015, and June 30, 2019 (period 2), reflecting approval and broader availability of BRAF and MEK inhibitor combination therapy and anti-PD-1/programmed death ligand 1 therapies alone or in combination with anti-CTLA-4 therapy.

HCRU and health care costs incurred from the first diagnosis of metastatic melanoma to the end of follow-up were quantified and compared at patient level between period 1 and period 2. If a patient was identified in both periods, the patient was assigned to period 1 (when first diagnosis of metastatic melanoma occurred) to ensure mutually exclusive groups for comparison in period 1 vs period 2 .

Categorical outcomes were summarized using counts and percentages and were compared between cohorts using chi-square tests for independence. Continuous outcomes were summarized using means and SDs and were compared using Wilcoxon rank-sum tests. 


\section{FIGURE 1 Patient Attrition}

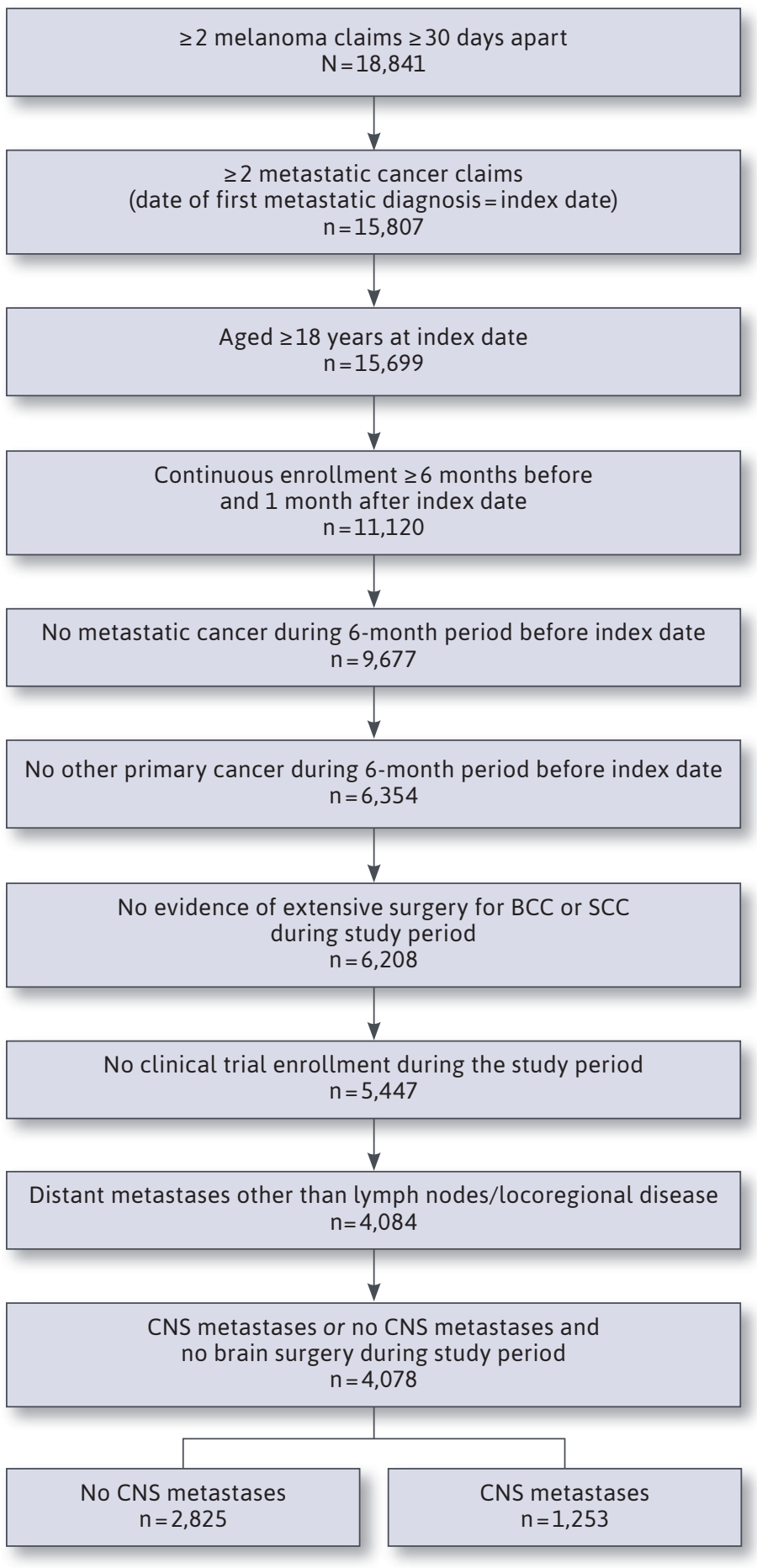

$\mathrm{BCC}=$ basal cell carcinoma; $C N S=$ central nervous system; $S C C=$ squamous cell carcinoma.

\section{Results}

\section{PATIENTS}

After applying inclusion and exclusion criteria, 4,078 patients with metastatic melanoma were included in the analysis (Figure 1). A total of 1,253 patients (30.7\%) had a diagnosis of CNS metastases, of whom 527 (42.1\%) had CNS metastases at the time of first metastatic diagnosis. Patient demographics were generally similar between patients with vs without CNS metastases, including age (median 58 years [interquartile range $\{\mathrm{IQR}\}=51-63]$ vs 58 years $[\mathrm{IQR}=49-54]$ ); male sex (62.9\% vs 58.8\%); and Charlson Comorbidity Index score (median $0.0[\mathrm{IQR}=0.0-1.0]$ vs $0.0[\mathrm{IQR}=0.0-1.0]$ ). The majority of patients had commercial insurance $(61.1 \%$ vs $58.3 \%$ ) or were self-insured (33.4\% vs $36.0 \%$; Supplementary Table 1, available in online article).

The overall median follow-up time was 15.1 months (IQR=7.4-28.5). Median follow-up time was significantly shorter for patients with CNS metastases than for patients without CNS metastases (11.1 months [IQR $=5.4-21.7]$ vs 17.1 months $[\mathrm{IQR}=8.6-31.4]$, respectively; $\mathrm{P}<0.001)$. Median time from the date of first diagnosis of metastatic melanoma to the first diagnosis of CNS metastases was 0.5 months $(\mathrm{IQR}=0.0-6.8)$ and was significantly shorter for patients with a first diagnosis of metastatic melanoma in 2015-2019 compared with those with a first diagnosis in 2011-2014 (0.4 months $[\mathrm{IQR}=0.0-5.9]$ vs 0.9 months $[\mathrm{IQR}=0.0-7.6]$, respectively; $P=0.04$ ).

Median time to first diagnosis of CNS metastases for patients diagnosed after the date of first diagnosis of metastatic melanoma was 5.5 months (IQR=1.2-11.3) and was also significantly shorter for patients with a first diagnosis in 2015-2019 vs 2011-2014 (4.6 months [IQR=0.8-11.2] vs 6.0 months $[\mathrm{IQR}=1.9-11.5]$, respectively; $\mathrm{P}=0.009$ ).

\section{HEALTH CARE RESOURCE UTILIZATION}

HCRU, 2011-2019. HCRU was higher in patients with CNS metastases than in those without CNS metastases across all categories assessed (Table 1). Patients with CNS metastases were more likely to receive any treatment for melanoma than those without CNS metastases (89.1\% vs $58.9 \%$; $\mathrm{P}<0.001)$, including systemic treatment $(73.3 \%$ vs $55.4 \% ; \mathrm{P}<0.001)$ and radiation treatment $(65.8 \%$ vs $11.8 \%$; $P<0.001)$, respectively. Compared with patients without CNS metastases, those with CNS metastases had more hospitalizations (mean [SD] 2.69 [2.56] vs 1.03 [1.64]; median 2.00 [IQR=1.00-4.00] vs $0.00[\mathrm{IQR}=0.00-1.00] ; \mathrm{P}<0.001)$ and emergency department visits (mean [SD] 1.80 [2.19] vs 0.95 [1.81]; median $1.00[\mathrm{IQR}=0.00-3.00]$ vs $0.00[\mathrm{IQR}=0.00-1.00] ; \mathrm{P}<0.001$, respectively. 


\section{TABLE 1 Health Care Resource Utilization During the Variable Post-Index Follow-Up Period for Patients Diagnosed With Metastatic Melanoma Between 2011 and 2019}

\begin{tabular}{|c|c|c|c|c|c|}
\hline HCRU & \multicolumn{2}{|c|}{ No CNS metastases, $n=2,825$} & \multicolumn{2}{|c|}{ CNS metastases, $n=1,253$} & $P$ value \\
\hline \multicolumn{6}{|l|}{ Number of hospitalizations } \\
\hline Mean (SD) & 1.03 & $(1.64)$ & 2.69 & $(2.56)$ & \multirow{2}{*}{$<0.001$} \\
\hline Median (IQR) & 0.00 & $(0.00-1.00)$ & 1.00 & $(0.00-4.00)$ & \\
\hline \multicolumn{6}{|l|}{ Number of ED visits } \\
\hline Mean (SD) & 0.95 & $(1.81)$ & 1.80 & $(2.19)$ & \multirow{2}{*}{$<0.001$} \\
\hline Median (IQR) & 0.00 & $(0.00-1.00)$ & 1.00 & $(0.00-3.00)$ & \\
\hline Any melanoma treatment, $\mathrm{n}(\%)^{\mathrm{a}}$ & 1,663 & $(58.9)$ & 1,117 & $(89.1)$ & $<0.001$ \\
\hline Chemotherapy, n (\%) & 1,458 & $(51.6)$ & 745 & $(59.5)$ & $<0.001$ \\
\hline Immunotherapy, n (\%) & 938 & $(33.2)$ & 579 & $(46.2)$ & $<0.001$ \\
\hline Targeted therapy, $\mathbf{n}(\%)^{d}$ & 241 & $(8.5)$ & 378 & $(30.2)$ & $<0.001$ \\
\hline Any systemic anticancer treatment, $\mathrm{n}(\%)$ & 1,564 & $(55.4)$ & 918 & $(73.3)$ & $<0.001$ \\
\hline Radiation therapy, n (\%) & 333 & $(11.8)$ & 825 & $(65.8)$ & $<0.001$ \\
\hline CNS surgery, n (\%) & & 0 & 347 & $(27.7)$ & $<0.001$ \\
\hline \multicolumn{6}{|l|}{ Dexamethasone 4 mg, n (\%) } \\
\hline Within \pm 30 days of metastatic diagnosis date & 44 & $(1.6)$ & 294 & $(23.5)$ & $<0.001$ \\
\hline Within \pm 30 days of CNS metastases diagnosis date & & - & 501 & $(40.0)$ & - \\
\hline \multicolumn{6}{|c|}{ Brain imaging any time after metastatic diagnosis date, $n(\%)$} \\
\hline $\mathrm{MRI} / \mathrm{CT}$ & 1,898 & $(67.2)$ & 1,232 & $(98.3)$ & $<0.001$ \\
\hline MRI & 1,688 & $(59.8)$ & 1,181 & $(94.3)$ & $<0.001$ \\
\hline $\mathrm{CT}$ & 617 & $(21.8)$ & 862 & $(68.8)$ & $<0.001$ \\
\hline \multicolumn{6}{|c|}{ Brain imaging within \pm 30 days of metastatic diagnosis date, $n(\%)$} \\
\hline $\mathrm{MRI} / \mathrm{CT}$ & 1,107 & $(39.2)$ & 894 & $(71.3)$ & $<0.001$ \\
\hline MRI & 991 & $(35.1)$ & 825 & $(65.8)$ & $<0.001$ \\
\hline $\mathrm{CT}$ & 159 & $(5.6)$ & 422 & $(33.7)$ & $<0.001$ \\
\hline \multicolumn{6}{|c|}{ Brain imaging any time after CNS metastases diagnosis date, $n$ (\%) } \\
\hline $\mathrm{MRI} / \mathrm{CT}$ & & - & 1,185 & $(94.6)$ & - \\
\hline MRI & & - & 1,122 & $(89.5)$ & - \\
\hline $\mathrm{CT}$ & & - & 808 & $(64.5)$ & - \\
\hline \multicolumn{6}{|c|}{ Brain imaging within \pm 30 days of CNS metastases diagnosis date, $n(\%)$} \\
\hline $\mathrm{MRI} / \mathrm{CT}$ & & - & 1,160 & $(92.6)$ & - \\
\hline MRI & & - & 1,090 & (87.0) & - \\
\hline $\mathrm{CT}$ & & - & 610 & $(48.7)$ & - \\
\hline
\end{tabular}

a Melanoma treatments included systemic chemotherapy; systemic targeted therapy; systemic immunotherapy; radiation therapy (stereotactic radiosurgery, brain radiation, or other radiation therapy); and brain surgery (including craniotomy).

${ }^{b}$ Chemotherapy included general chemotherapy administration CPT, ICD-9/10-CM procedure codes; specific chemotherapy drugs (azacitidine, bendamustine, cabazitaxel, capecitabine, carboplatin, chlorambucil, cladribine, cisplatin, dacarbazine, doxorubicin, enzalutamide, fludarabine, fluorouracil, gemcitabine, ifosfamide, irinotecan, lenalidomide, lomustine, oxaliplatin, paclitaxel, temozolomide, thalidomide, vinblastine, and vinorelbine); or other (bevacizumab, interferon-alfa-2b).

Immunotherapy included nivolumab, pembrolizumab, and ipilimumab.

${ }^{\top}$ Targeted therapy included dabrafenib, trametinib, vemurafenib, cobimetinib, encorafenib, binimetinib, and imatinib.

CNS = central nervous system; CPT =Current Procedural Terminology; $C T=$ computed tomography; $E D=$ emergency department; ICD-9/10-CM =International Classification of Diseases, Ninth/Tenth Revision, Clinical Modification; IQR=interquartile range; $M R I=$ magnetic resonance imaging. 


\section{FIGURE 2 Mean PPPM Overall Costs in the Variable Post-Index Follow-Up Period for Patients Diagnosed With Metastatic Melanoma Between 2011 and 2019}

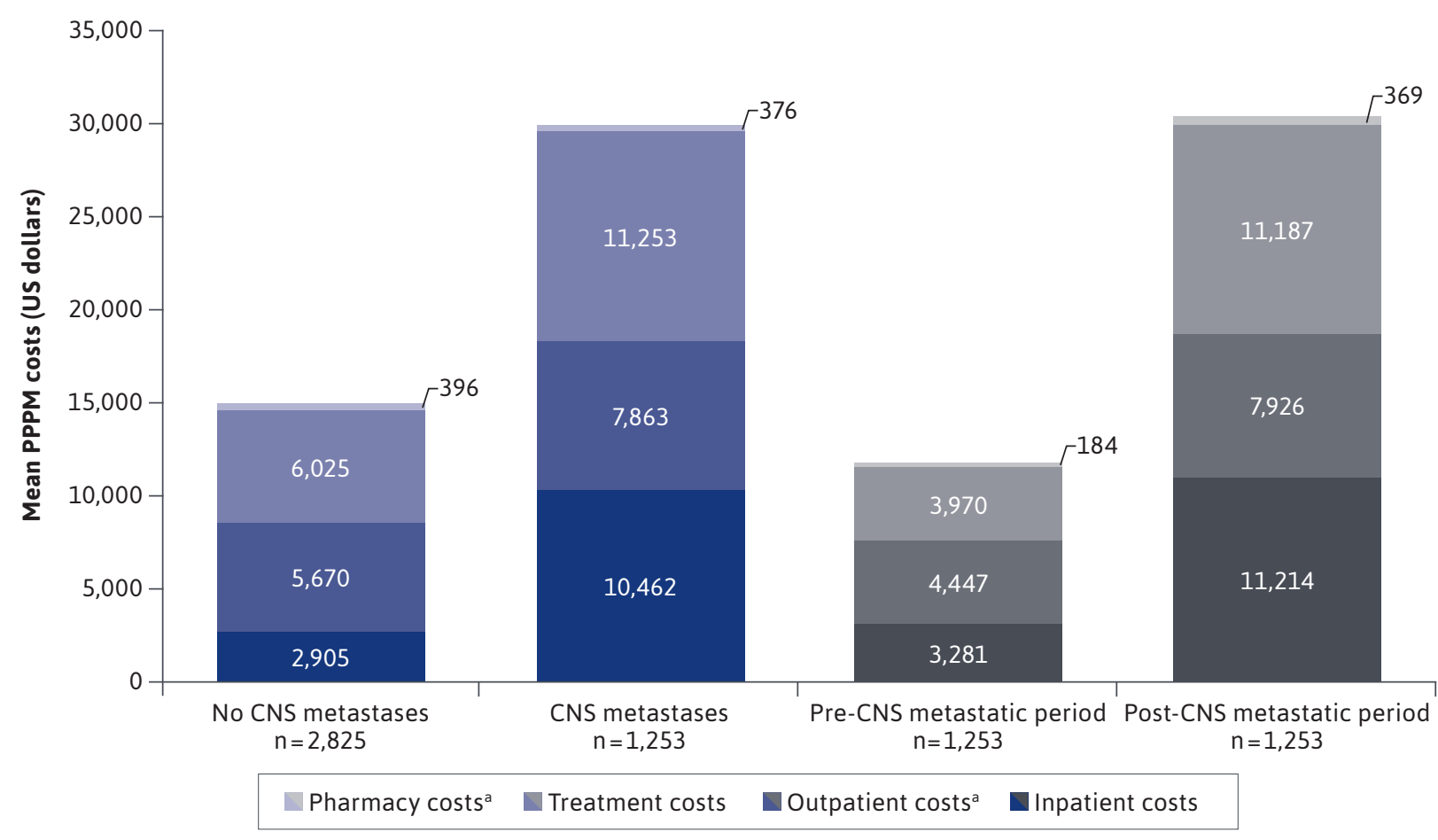

aEcluding costs of melanoma treatments.

CNS = central nervous system; PPPM = per patient per month.

Use of brain MRI/CT imaging any time after metastatic diagnosis (98.3\% vs $67.2 \%$; $\mathrm{P}<0.001)$ and within plus or minus 30 days of the first metastatic diagnosis date $(71.3 \%$ vs $39.2 \%$; $P<0.001)$ was higher in patients with vs without CNS metastases (Table 1), respectively. MRI was the most common imaging modality in both cohorts $(94.3 \%$ vs $59.8 \%$ in patients with vs without CNS metastases; P<0.001), whereas CT was used less frequently (68.8\% vs 21.8\%; $\mathrm{P}<0.001)$.

The proportion of patients with claims for dexamethasone $4 \mathrm{mg}$ within plus or minus 30 days of metastatic diagnosis date was higher in patients with CNS metastases than in those without CNS metastases $(23.5 \%$ vs $1.6 \%$, respectively; $\mathrm{P}<0.001$; Table 1). In patients with CNS metastases, $40.0 \%$ had claims for dexamethasone $4 \mathrm{mg}$ within plus or minus 30 days of CNS metastatic diagnosis date.

HCRU, 2011-2014 vs 2015-2019. Patients with CNS metastases had significantly greater HCRU than patients without CNS metastases regardless of time period of first diagnosis of metastatic melanoma (all $\mathrm{P}<0.001$; Supplementary Table 2, available in online article). Patterns of HCRU changed over time from 2011-2014 to 2015-2019 in patients with CNS metastases (Supplementary Table 3 , available in online article). In patients with CNS metastases, the proportion of patients with claims for any systemic treatment increased from $64.5 \%$ among those with a first diagnosis of metastatic melanoma in 2011-2014 to $81.2 \%$ among those with a first diagnosis in 2015-2019 $(\mathrm{P}<0.001)$.

Compared with patients with a first diagnosis of metastatic melanoma in 2011-2014, those with a first diagnosis in 2015-2019 were more likely to have received chemotherapy (68.1\% vs 50.0\%; P<0.001), immunotherapy $(60.9 \%$ vs $30.1 \%$; $P<0.001)$, and/or targeted therapies $(32.7 \%$ vs $27.4 \%$; $P=0.05$ ), respectively. The mean number of hospitalizations per patient decreased slightly in 2015-2019 vs 2011-2014 (mean [SD] 2.46 [2.38] vs 2.94 [2.73]; median 2.00 [IQR=1.00-4.00] vs 2.00 [IQR=1.00-4.00]; $\mathrm{P}<0.001$ ), whereas the mean number of emergency department visits per patient increased slightly (mean [SD] 1.92 [2.28] vs 1.67 


\section{FIGURE 3 Mean PPPM Outpatient Costs in the Variable Post-Index Follow-Up Period for Patients Diagnosed With Metastatic Melanoma Between 2011 and 2019}

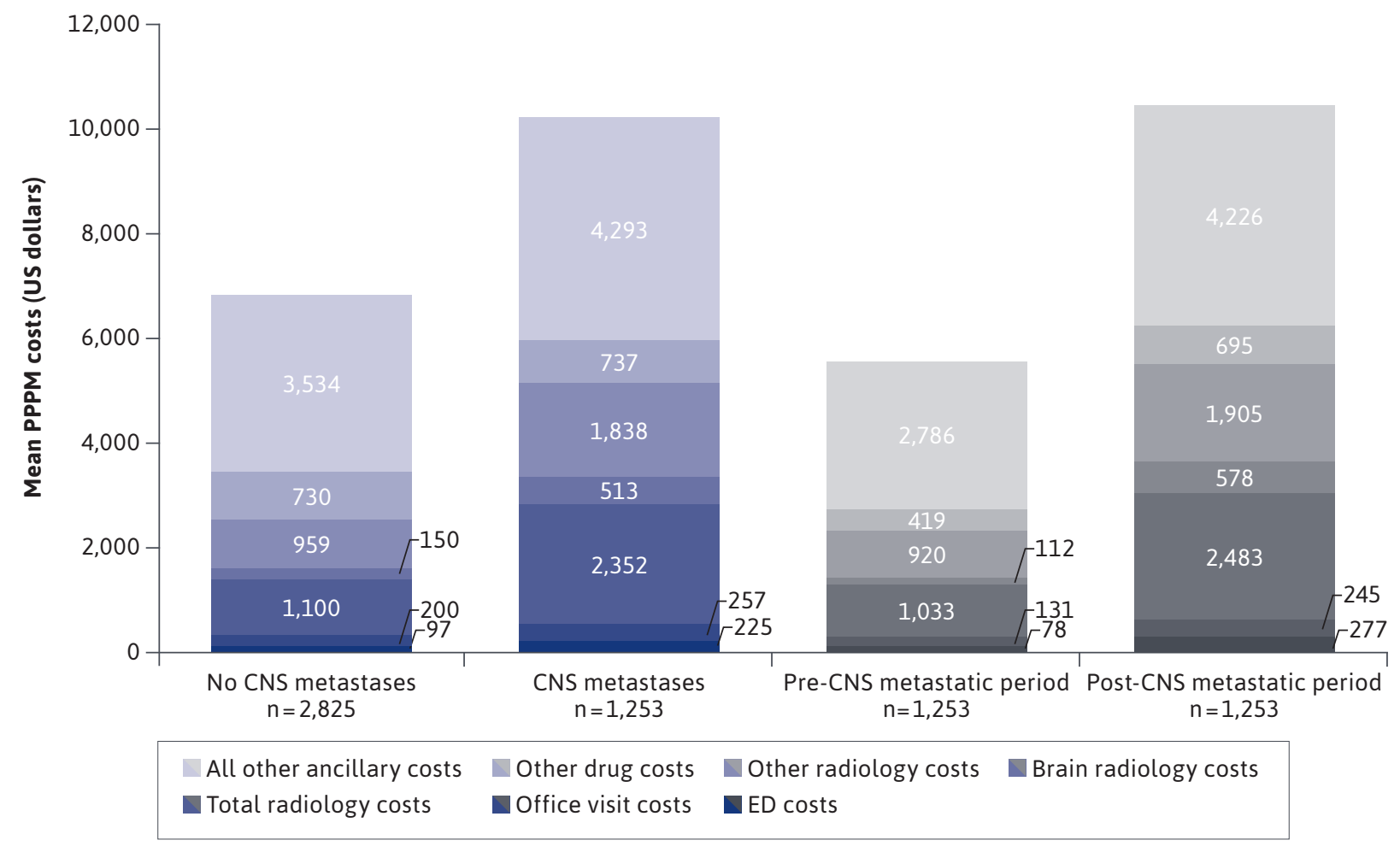

$C N S=$ central nervous system; $E D=$ emergency department PPPM = per patient per month .

[2.09]; median $1.00[\mathrm{IQR}=0.00-3.00]$ vs $1.00[\mathrm{IQR}=0.00-$ 2.00]; $P=0.06)$.

Although a similar proportion of patients had MRI/CT imaging any time after metastatic diagnosis $(98.5 \%$ in $2015-$ 2019 vs $98.2 \%$ in 2011-2014) or within plus or minus 30 days of the first metastatic diagnosis date $72.8 \%$ in $2015-2019$ vs $69.7 \%$ in 2011-2014), the number of MRI/CT scans per patient increased in 2015-2019 vs 2011-2014 (mean [SD] 7.18 [5.45] vs 6.21 [4.63]; median 6.00 [IQR=3.00-10.00] vs 5.00 $[\mathrm{IQR}=3.00-8.00] ; \mathrm{P}=0.002)$.

\section{COSTS}

Health Care Costs, 2011-2019. Patients with CNS metastases incurred higher total PPPM costs than those without CNS metastases (mean [SD] $\$ 29,953$ [\$25,598] vs $\$ 14,996$ [\$21,204]; median $\$ 23,631.45 \quad$ [IQR $=\$ 12,265-\$ 40,410.56]$ vs $\$ 8,849.03 \quad[\mathrm{IQR}=\$ 3,044.05-\$ 19,301.64] ; \quad \mathrm{P}<0.001$; Figure 2). Higher total PPPM costs were incurred in the post-CNS metastatic period compared with the pre-CNS metastatic period (\$30,696 [\$26,476] vs. \$11,883 [\$30,376]; median $\$ 24,347.54[\mathrm{IQR}=\$ 12,031.07-\$ 41,232.66]$ vs. $\$ 0.00$ $[\mathrm{IQR}=\$ 0.00-\$ 13,400.56] ; \mathrm{P}<0.001)$. All outpatient costs, except for nonmelanoma outpatient drug costs, were significantly higher for patients with CNS metastases compared with those without CNS metastases (all $\mathrm{P}<0.001$; Figure 3). Costs related to all outpatient categories were significantly higher in the post-CNS metastatic period than in the preCNS metastatic period (all $\mathrm{P}<0.001$ ).

Mean treatment costs for targeted therapy, immunotherapy, chemotherapy, radiation therapy, and outpatient brain surgical procedures were significantly higher for patients with CNS metastases than for those without CNS metastases (all $\mathrm{P}<0.001$; Figure 4). In patients with $\mathrm{CNS}$ metastases, mean treatment costs were significantly higher in the post-CNS metastatic period than in the pre-CNS metastatic period (all $\mathrm{P}<0.001)$.

Health Care Costs, 2011-2014 vs 2015-2019. Patients with CNS metastases had significantly greater costs than 


\section{FIGURE 4 Mean PPPM Treatment Costs in the Variable Post-Index Follow-Up Period for Patients Diagnosed With Metastatic Melanoma Between 2011 and 2019}

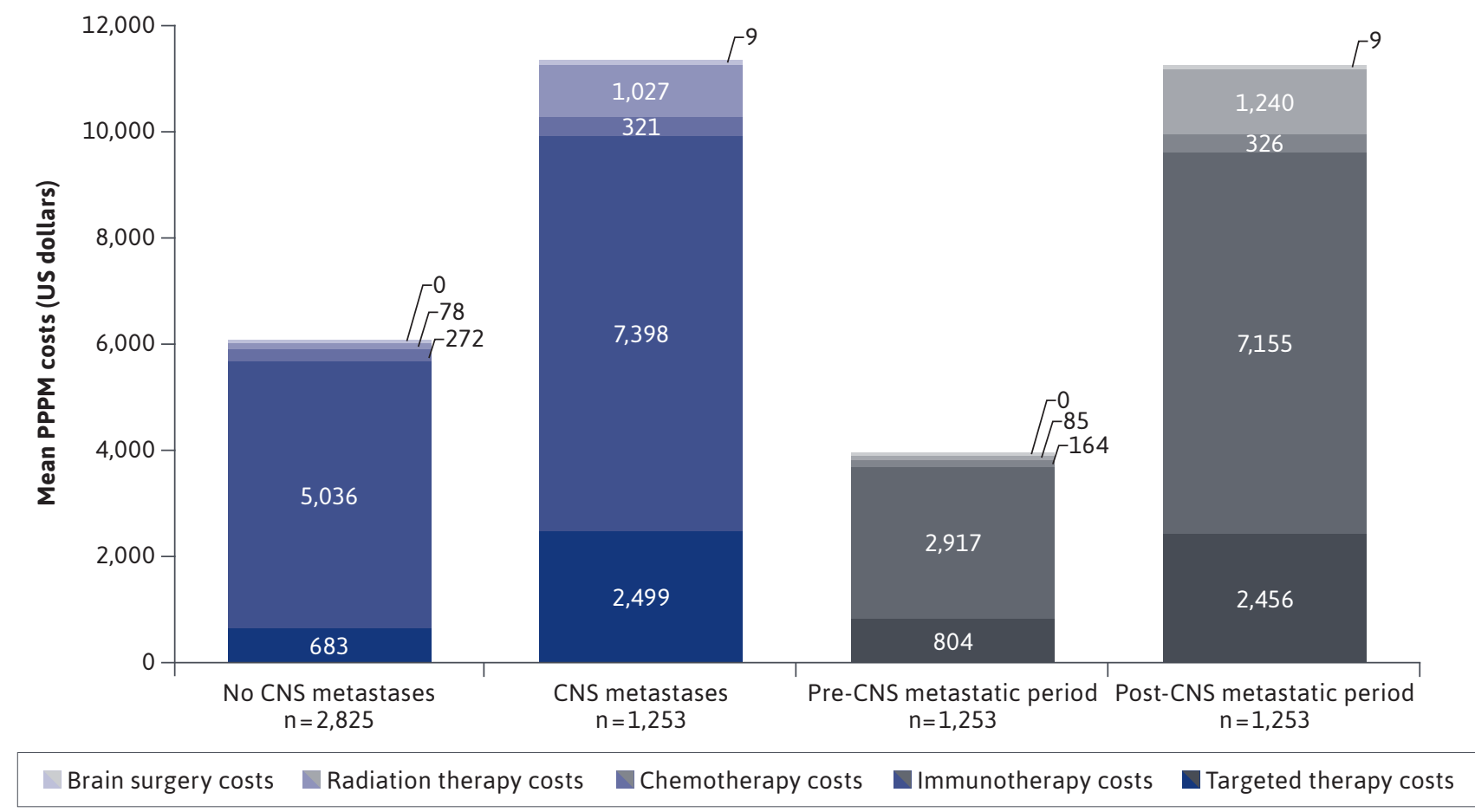

CNS = central nervous system; PPPM= per patient per month.

patients without CNS metastases regardless of time period of first diagnosis of metastatic melanoma (Supplementary Table 4, available in online article).

In patients with CNS metastases, mean (SD) total PPPM costs increased from $\$ 28,183$ (\$25,820; median $\$ 20,971.04$ $[\mathrm{IQR}=\$ 10,055.87-\$ 38,341.93])$ in patients with a first diagnosis of metastatic melanoma in 2011-2014 to $\$ 31,569$ (\$25,306; median $\$ 25,762.85[\mathrm{IQR}=\$ 14,412.80-\$ 41,431.09])$ in those with a first diagnosis in 2015-2019 $(\mathrm{P}<0.001$; Supplementary Figure 2, available in online article). The main drivers of increased costs were increases in costs associated with immunotherapies and targeted therapies.

In patients with CNS metastases, mean (SD) PPPM treatment costs increased from $\$ 8,173$ (\$12,933; median $\$ 2,702.67[\mathrm{IQR}=\$ 207.42-\$ 11,097.74])$ in $2011-2014$ to $\$ 14,065$ (\$14,909; median $\$ 10,806.35$ [IQR=\$2,599.34-\$20,951.49]) in 2015-2019 $(\mathrm{P}<0.001$; Supplementary Figure 3, available in online article). From 2011-2004 to 2015-2019, immunotherapy costs (SD) increased from $\$ 5,190$ (\$12,050; median
$\$ 0.00[\mathrm{IQR}=\$ 0.00-\$ 5,079.51])$ to $\$ 9,414(\$ 14,022 ;$ median $\$ 3,487.17$ [IQR $=\$ 0.00-\$ 13,595.18] ; P<0.001)$, and targeted therapy costs increased from $\$ 1,703(\$ 3,590$; median $\$ 0.00$ $[\mathrm{IQR}=\$ 0.00-\$ 1,043.05])$ to $\$ 3,226(\$ 5,812 ;$ median $\$ 0.00$ $[\mathrm{IQR}=\$ 0.00-\$ 24,222.16] ; \mathrm{P}=0.002)$, respectively.

Mean (SD) chemotherapy costs decreased significantly from $\$ 397(\$ 1,018)$ to $\$ 251$ (\$449), although median costs showed a significant increase from $\$ 0.00 \quad(\mathrm{IQR}=\$ 0.00-$ $\$ 257.28)$ to $\$ 107.54$ (IQR $=\$ 0.00-\$ 334.07$; both $\mathrm{P}<0.001)$. Radiation therapy costs were numerically increased from $\$ 873$ (\$1,700; median \$251.16 [IQR $=\$ 0.00-\$ 964.33])$ in 2011-2014 to $\$ 1,167$ ( $\$ 2,175 ;$ median $\$ 296.86$ [IQR=\$0.00$\$ 1,336.24])$ in 2015-2019 ( $\mathrm{P}=0.26)$. Mean (SD) PPPM inpatient costs decreased from $\$ 11,240$ (\$17,603; median $\$ 4,824.16$ $[\mathrm{IQR}=\$ 1,474.07-\$ 12,862.46])$ in $2011-2014$ to $\$ 9,751(\$ 16,275$; median $\$ 3,887.70 \quad[\mathrm{IQR}=\$ 775.73-\$ 10,378.95])$ in $2015-2019$ $(P=0.009)$, whereas outpatient costs were largely unchanged (Supplementary Figure 4, available in online article). 


\section{Discussion}

Data from this real-world study demonstrate that a considerable proportion of patients with melanoma continue to develop CNS metastases despite significant advances in systemic treatment. With a median follow-up of 15 months in the current study, 31\% of patients had developed CNS metastases, consistent with previously published estimates. ${ }^{5,37}$

Patients with CNS metastases incurred significantly higher HCRU and costs compared with those without CNS metastases. These costs were attributable to treatment of CNS metastases and were primarily incurred after diagnosis of CNS metastasis. In patients with CNS metastases, costs incurred before diagnosis of CNS metastases were similar to those incurred in patients without CNS metastases. For most categories, costs were more than doubled in the period after diagnosis of CNS metastases compared with the period before diagnosis. Costs were significantly higher for patients with CNS metastases across inpatient, outpatient, treatment, and pharmacy services.

For patients with CNS metastases, the largest contributors to total costs were total radiology, targeted therapies, and immunotherapies. Among patients with metastatic melanoma diagnosed in 2015-2019, use of any systemic therapy, particularly immunotherapy, increased compared with those diagnosed in 2011-2014, reflecting increased availability of effective systemic treatment options. ${ }^{15-25}$

Concomitantly, patients diagnosed in the later time period had increased treatment costs related to immunotherapy and targeted therapy, although this was partially offset by reduced costs related to inpatient treatment, which may reflect better tumor control with these modern treatments, ${ }^{15-25}$ resulting in fewer complications (such as seizures and intracranial hemorrhages) that require hospitalization and/or surgical intervention. Indeed, we have reported improved overall survival outcomes for patients with melanoma and CNS metastases over time, particularly for those treated with stereotactic radiosurgery alone or in combination with systemic therapy (1-year overall survival rate, $69 \%$ in $2015-2018$ vs $53 \%$ in $2011-2014)$, using real-world data from the nationwide, longitudinal Flatiron Health database. ${ }^{38}$

As previously noted, multiple randomized controlled trials have demonstrated improved survival outcomes compared with previous standards of care for targeted therapies and immunotherapies in patients with metastatic melanoma. ${ }^{15-25}$ Despite high treatment costs, cost-effectiveness has been established for anti-PD-1 therapies (nivolumab and pembrolizumab) in metastatic melanoma, ${ }^{39}$ but patients with CNS metastases were generally excluded from these studies. Stereotactic radiosurgery is recommended as a preferred treatment strategy for CNS metastases in patients with melanoma and has been demonstrated to be cost-effective under certain conditions. ${ }^{8,40}$ Given the limited clinical data evaluating use of stereotactic radiosurgery in combination with immunotherapy, further evaluation of the clinical benefit and associated medical value is required.

CNS involvement can cause debilitating neurological symptoms, and patients with symptomatic CNS metastases have worse treatment outcomes and prognosis than patients with asymptomatic CNS metastases. ${ }^{2}$ Among patients with CNS metastases in the current study, a significant proportion (40\%) received treatment with dexamethasone $4 \mathrm{mg}$ within 30 days of diagnosis, indicative of the presence of symptomatic CNS metastases at the time of diagnosis. ${ }^{34}$ Pivotal studies of immune checkpoint inhibitors in melanoma largely excluded patients with CNS metastases, ${ }^{23-25}$ and studies evaluating the intracranial activity of these agents have generally been limited to asymptomatic patients not receiving concomitant corticosteroids. ${ }^{26-29}$

Corticosteroids may antagonize the effects of immunotherapy via their immunosuppressive activity, ${ }^{35}$ and studies in patients with melanoma or non-small cell lung cancer with CNS metastases have suggested that patients receiving concomitant corticosteroids have worse outcomes on immune checkpoint inhibitor therapy than those not receiving concomitant corticosteroids. ${ }^{26,29,41,42}$ However, it remains unclear whether corticosteroids compromise the efficacy of immune checkpoint inhibitors or if patients receiving concomitant corticosteroids merely represent a patient population with worse prognosis. These patients represent an unmet medical need due to limited responsiveness to currently available treatment options, including targeted therapies and immunotherapy therapies. ${ }^{26-30}$

Given the high incidence of CNS metastases in patients with metastatic melanoma, current guidelines have recommended brain MRI/CT imaging as part of the staging process at the time of diagnosis of metastatic disease since $2013 .^{7,8}$ However, fewer than $40 \%$ of patients without CNS metastases underwent brain imaging within 30 days of the diagnosis of metastatic disease across the entire study period (2011-2019), increasing from 36\% in 2011-2014 to $41 \%$ in $2015-2019$. Interestingly, the median time from first metastatic diagnosis to diagnosis of CNS metastases was shorter in 2015-2019 (4.6 months) compared with 2011-2014 (6.0 months), which may reflect increased use of brain imaging for restaging and surveillance in patients with metastatic melanoma over time, as observed in the current study.

The relatively short interval between baseline metastatic assessment and the diagnosis of CNS metastasis suggests that many of these patients may have had asymptomatic 
CNS disease at the time of their initial staging assessment. These observations support the adoption of baseline screening for CNS metastasis into routine clinical practice. Given the improved outcomes for local and systemic treatments in patients with asymptomatic CNS metastases, as well as the limited corticosteroid use in this population, there appears to be an opportunity to improve outcomes through early detection of CNS metastases, potentially reducing the high HCRU and costs associated with CNS metastases.

\section{LIMITATIONS}

This study used insurance claims data to assess real-world HCRU and health care costs associated with CNS metastases in patients with metastatic melanoma. Thus, our study is subject to the inherent limitations of claims databases, including the potential for missing or inaccurate diagnostic or procedure codes. In particular, the presence of CNS metastatic diagnosis may be underreported in claims data, since some CNS metastases may be coded as secondary malignant neoplasms without specification of sites or misclassified with unspecified metastasis codes. Insurance claims may not accurately reflect actual treatment use because claims may be made for prescriptions that are filled but not used.

Additionally, the data did not capture any care that patients may have received for which no insurance claims were made. The IQVIA PharMetrics Plus database represents a relatively young population of commercially insured patients with a median age of 58 years at metastatic diagnosis compared with a median age at melanoma diagnosis of 65 years in the general population. ${ }^{43}$ Also, information on race/ethnicity, which may influence HCRU and costs, was not available in this dataset. Consequently, data from this study population may not be generalizable to all patients with metastatic melanoma in the United States.

Although HCRU may be expected to increase with longer follow-up durations, we did not adjust for differences in follow-up in our analyses. Nevertheless, we observed higher HCRU in patients with vs without CNS metastases, despite the potential for underestimation of HCRU in these patients as a result of the shorter follow-up duration.

In the absence of death data, it is unclear whether the shorter followup duration for patients with CNS metastases in this study reflects deaths or loss to follow-up for unknown reasons; however, the difference of approximately 6 months between cohorts appears consistent with known differences in survival outcomes between patients with vs without CNS metastases. ${ }^{5}$

\section{Conclusions}

This study demonstrates that CNS metastases occur frequently in patients with metastatic melanoma and are associated with significantly increased economic burden compared with patients without CNS metastases-the largest contributors to total costs in the current treatment era are radiology, targeted therapies, and immunotherapies. A substantial proportion of patients have symptomatic disease requiring treatment with corticosteroids, and these patients represent an unmet medical need because of limited responsiveness to currently available treatment options. Brain imaging remains underused and additional screening is needed to facilitate earlier diagnosis of asymptomatic CNS metastases when they might be more responsive to currently available therapeutic options.

\section{DISCLOSURES}

This study was funded by F. Hoffmann-La Roche Ltd. The sponsor was involved in the study design, data collection, data analysis, manuscript preparation, and publication decisions. Seetasith and Lee are employed by and report stock ownership in Genentech, Inc. Bartley and McKenna were employed by Genentech, Inc., at the time of this study and report stock ownership. Tawbi reports grants and personal fees from Genentech/Roche, Novartis, BMS, and Merck; grants from GSK and Celgene; and personal fees from Eisai, outside the submitted work. Kent, Burton, and Haydu have nothing to disclose.

The results of this study were presented in part at the AMCP Nexus 2020 Virtual Meeting, October 19-23, 2020.

\section{ACKNOWLEDGMENTS}

Medical writing and editorial support for this manuscript was provided by Melanie Sweetlove, MSc (ApotheCom, San Francisco, CA), and funded by F. Hoffmann-La Roche Ltd.

\section{REFERENCES}

1. Fife KM, Colman MH, Stevens GN, et al. Determinants of outcome in melanoma patients with cerebral metastases. J Clin Oncol. 2004;22(7):1293-300.

2. Cohen JV, Tawbi H, Margolin KA, et al. Melanoma central nervous system metastases: Current approaches, challenges, and opportunities. Pigment Cell Melanoma Res. 2016;29(6):627-42.

3. Zhang D, Wang Z, Shang D, et al. Incidence and prognosis of brain metastases in cutaneous melanoma patients: a population-based study. Melanoma Res. 2019;29(1):77-84.

4. Davies MA, Liu P, McIntyre S, et al. Prognostic factors for survival in melanoma patients with brain metastases. Cancer. 2011;117(8):1687-96.

5. Sadetsky N, Hernandez A, Wallick CJ, et al. Survival outcomes in an older US population with advanced melanoma and central nervous system metastases: SEER-Medicare analysis. Cancer Med. 2020;9(17):6216-24. 
6. Fink KR, Fink JR. Imaging of brain metastases. Surg Neurol Int. 2013;4 (Suppl 4):S209-19.

7. Gershenwald JE, Scolyer RA, Hess KR, et al. Melanoma staging: evidencebased changes in the American Joint Committee on Cancer eighth edition cancer staging manual. CA Cancer J Clin. 2017;67(6):472-92.

8. National Comprehensive Cancer Network ${ }^{\circledR}\left(\mathrm{NCCN}^{\circledR}\right)$ NCCN Clinical Practice Guidelines in Oncology. Cutaneous melanoma (Version 4.2020), 2020. Accessed October 26, 2020. https:// www.nccn.org/professionals/physician_ gls/pdf/cutaneous melanoma.pdf

9. Zukauskaite R, Schmidt H, Asmussen JT, et al. Asymptomatic brain metastases in patients with cutaneous metastatic malignant melanoma. Melanoma Res. 2013;23(1):21-26.

10. Brastianos HC, Nguyen P, Sahgal A, et al. Association of innovations in radiotherapy and systemic treatments with clinical outcomes in patients with melanoma brain metastasis from 2007 to 2016 . JAMA Netw Open. 2020;3(7):e208204.

11. Goyal S, Silk AW, Tian S, et al. Clinical management of multiple melanoma brain metastases: a systematic review. JAMA Oncol. 2015;1(5):668-76.

12. Suh JH, Kotecha R, Chao ST, et al. Current approaches to the management of brain metastases. Nat Rev Clin Oncol. 2020;17(5):279-99.

13. Aoyama H, Shirato H, Tago M, et al. Stereotactic radiosurgery plus wholebrain radiation therapy vs stereotactic radiosurgery alone for treatment of brain metastases: a randomized controlled trial. JAMA. 2006;295(21):2483-91.

14. Churilla TM, Ballman KV, Brown PD, et al. Stereotactic radiosurgery with or without whole-brain radiation therapy for limited brain metastases: a secondary analysis of the North Central Cancer Treatment Group N0574 (Alliance) randomized controlled trial. Int J Radiat Oncol Biol Phys. 2017;99(5):1173-78.
15. Bollag G, Tsai J, Zhang J, et al. Vemurafenib: the first drug approved for BRAF-mutant cancer. Nat Rev Drug Discov. 2012;11(11):873-86.

16. Chapman PB, Hauschild A, Robert C, et al. Improved survival with vemurafenib in melanoma with BRAF V600E mutation. N Engl J Med. 2011;364(26):2507-16.

17. Larkin J, Ascierto PA, Dreno B, et al. Combined vemurafenib and cobimetinib in BRAF-mutated melanoma. N Engl J Med. 2014;371(20):1867-76.

18. Hauschild A, Grob JJ, Demidov LV, et al. Dabrafenib in BRAF-mutated metastatic melanoma: A multicentre, open-label, phase 3 randomised controlled trial. Lancet. 2012;380(9839):358-65.

19. Queirolo P, Spagnolo F. BRAF plus MEK-targeted drugs: A new standard of treatment for BRAF-mutant advanced melanoma. Cancer Metastasis Rev. 2017;36(1):35-42.

20. Long GV, Stroyakovskiy D, Gogas H, et al. Combined BRAF and MEK inhibition versus BRAF inhibition alone in melanoma. N Engl J Med. 2014;371(20):1877-88.

21. Robert C, Karaszewska B, Schachter J, et al. Improved overall survival in melanoma with combined dabrafenib and trametinib. N Engl J Med. 2015;372(1):30-39.

22. Arasanz H, Lacalle A, Lecumberri MJ, et al. Immunotherapy in malignant melanoma: Recent approaches and new perspectives. Melanoma Manag. 2017;4(1):39-48.

23. Robert C, Schachter J, Long GV, et al. Pembrolizumab versus ipilimumab in advanced melanoma. N Engl J Med. 2015;372(26):2521-32.

24. Robert C, Long GV, Brady B, et al. Nivolumab in previously untreated melanoma without BRAF mutation. N Engl J Med. 2015;372(4):320-30.

25. Larkin J, Chiarion-Sileni V, Gonzalez R, et al. Combined nivolumab and ipilimumab or monotherapy in untreated melanoma. N Engl J Med. 2015;373(1):23-34.
26. Margolin K, Ernstoff MS, Hamid O, et al. Ipilimumab in patients with melanoma and brain metastases: an open-label, phase 2 trial. Lancet Oncol. 2012;13(5):459-65.

27. Tawbi HA, Forsyth PA, Algazi A, et al. Combined nivolumab and ipilimumab in melanoma metastatic to the brain. N Engl J Med. 2018;379(8):722-30.

28. Long GV, Atkinson V, Lo S, et al. Combination nivolumab and ipilimumab or nivolumab alone in melanoma brain metastases: a multicentre randomised phase 2 study. Lancet Oncol. 2018;19(5):672-81.

29. Tawbi HA, Forsyth PA, Hodi FS, et al. Safety and efficacy of the combination of nivolumab plus ipilimumab in patients with melanoma and asymptomatic or symptomatic brain metastases (CheckMate 204). Neuro Oncol. 2021;23(11):1961-73.

30. Davies MA, Saiag P, Robert C, et al. Dabrafenib plus trametinib in patients with BRAF(V600)-mutant melanoma brain metastases (COMBI-MB): a multicentre, multicohort, open-label, phase 2 trial. Lancet Oncol. 2017;18(7):863-73.

31. Ahmed KA, Abuodeh YA, Echevarria MI, et al. Clinical outcomes of melanoma brain metastases treated with stereotactic radiosurgery and anti-PD-1 therapy, anti-CTLA-4 therapy, BRAF/ MEK inhibitors, BRAF inhibitor, or conventional chemotherapy. Ann Oncol. 2016;27(12):2288-94.

32. McArthur GA, Maio M, Arance A, et al. Vemurafenib in metastatic melanoma patients with brain metastases: an openlabel, single-arm, phase 2, multicentre study. Ann Oncol. 2017;28(3):634-41.

33. Geukes Foppen MH, Boogerd W, Blank CU, et al. Clinical and radiological response of BRAF inhibition and MEK inhibition in patients with brain metastases from BRAF-mutated melanoma. Melanoma Res. 2018;28(2):126-33. 
34. Ryken TC, Kuo JS, Prabhu RS, et al. Congress of Neurological Surgeons systematic review and evidence-based guidelines on the role of steroids in the treatment of adults with metastatic brain tumors. Neurosurgery. 2019;84(3):E189-91.

35. Garant A, Guilbault C, Ekmekjian T, et al. Concomitant use of corticosteroids and immune checkpoint inhibitors in patients with hematologic or solid neoplasms: a systematic review. Crit Rev Oncol Hematol. 2017;120:86-92.

36. Vekeman F, Cloutier M, Yermakov S, et al. Economic burden of brain metastases among patients with metastatic melanoma in a USA managed care population. Melanoma Res. 2014;24(6):602-10.

37. Chiarion-Sileni V, Guida M, Ridolfi L, et al. Central nervous system failure in melanoma patients: results of a randomised, multicentre phase 3 study of temozolomide-and dacarbazine-based regimens. Br J Cancer. 2011;104(12):1816-21.
38. Tawbi H, To TM, Wu N, et al. Treatment patterns and clinical outcomes for cutaneous melanoma patients with central nervous system metastases: a real-world study. Poster presented at: SMR 2019; November 20-23, 2019; Salt Lake City, UT. Accessed January 31, 2022. https://medically.gene.com/global/en/ asset-viewer.dfc9a86a-9007-4e07-ba30e603210f3b0d-restricted.html

39. Gorry C, McCullagh L, Barry M. Economic evaluation of systemic treatments for advanced melanoma: a systematic review. Value Health. 2020;23(1):52-60.

40. Lester-Coll NH, Dosoretz AP, Magnuson WJ, et al. Cost-effectiveness of stereotactic radiosurgery versus wholebrain radiation therapy for up to 10 brain metastases. J Neurosurg. 2016;125(Suppl 1):18-25.
41. Arbour KC, Mezquita L, Long N, et al. Impact of baseline steroids on efficacy of programmed cell death-1 and programmed death-ligand 1 blockade in patients with non-small-cell lung cancer. J Clin Oncol. 2018;36(28):2872-78.

42. Scott SC, Pennell NA. Early use of systemic corticosteroids in patients with advanced NSCLC treated with nivolumab. J Thoracic Oncol. 2018;13(11):1771-75.

43. Howlader N, Noone AM, Krapcho M, et al. SEER cancer statistics review, 19752018. National Cancer Institute. April 15, 2021. Accessed October 1, 2021. https:// seer.cancer.gov/csr/1975_2018/ 